\title{
Existence of solutions of first-order differential equations via a fixed point theorem for discontinuous operators
}

\author{
Rubén Figueroa Sestelo and Rodrigo López Pouso*
}

\section{"Correspondence:}

rodrigo.lopez@usc.es

Departamento de Análise

Matemática, Facultade de

Matemáticas, Universidade de

Santiago de Compostela, Campus

Sur, Santiago de Compostela,

15782, Spain

\begin{abstract}
We use a recent Schauder-type result for discontinuous operators in order to look for solutions for first-order differential equations subject to initial functional conditions. We show how this abstract fixed-point result allows us to consider a nonlinearity which can be strongly discontinuous. Some examples of applications and comparison with recent literature are included.
\end{abstract}

\section{Introduction and preliminaries}

In this paper we are concerned with the existence of absolutely continuous solutions of the initial value problem

$$
x^{\prime}=f(t, x) \quad \text { for a.a. } t \in I=\left[t_{0}-L, t_{0}+L\right], \quad x\left(t_{0}\right)=F(x)
$$

We assume that $t_{0} \in \mathbb{R}$ and $L>0$ are given (so (1.1) is a nonlocal problem), and $f: I \times \mathbb{R} \longrightarrow$ $\mathbb{R}$ is a not necessarily continuous function, satisfying some assumptions to be detailed. Finally, $F: \mathcal{C}(I) \longrightarrow \mathbb{R}$ is assumed to be continuous, but not necessarily linear or bounded. Notice that, under this framework, classical initial and multipoint conditions are included.

Our goal is to show that the following general version of Schauder's theorem proven in [1] can be employed to prove the existence of solutions of (1.1) under very general conditions.

Theorem 1.1 ([1], Theorem 3.1) Let $K$ be a nonempty, convex, and compact subset of a normed space $X$.

Any mapping $T: K \longrightarrow K$ has at least one fixed point provided that for every $x \in K$ we have

$$
\{x\} \cap \bigcap_{\varepsilon>0} \overline{\operatorname{co}} T\left(B_{\varepsilon}(x) \cap K\right) \subset\{T x\}
$$

where $B_{\varepsilon}(x)$ stands for the closed ball in $X$ with center $x$ and radius $\varepsilon>0$, and $\overline{c o}$ denotes the closed convex hull.

(c) 2015 Sestelo and Pouso. This article is distributed under the terms of the Creative Commons Attribution 4.0 International License (http://creativecommons.org/licenses/by/4.0/), which permits unrestricted use, distribution, and reproduction in any medium, provided you give appropriate credit to the original author(s) and the source, provide a link to the Creative Commons license, and indicate if changes were made. 
Basically, the use of Theorem 1.1 instead of the classical Schauder's theorem allows $f$ to be discontinuous over the graphs of countably many functions in the conditions of the following definition. Readers are referred to [2-4] for similar definitions.

Definition 1.1 An admissible discontinuity curve for the differential equation $x^{\prime}=f(t, x)$ is an absolutely continuous function $\gamma:[a, b] \subset I \longrightarrow \mathbb{R}$ satisfying one of the following conditions:

either $\gamma^{\prime}(t)=f(t, \gamma(t))$ for a.a. $t \in[a, b]$ (and we then say that $\gamma$ is viable for the

differential equation),

or there exist $\varepsilon>0$ and $\psi \in L^{1}(a, b), \psi(t)>0$ for a.a. $t \in[a, b]$, such that

either

$$
\gamma^{\prime}(t)+\psi(t)<f(t, y) \quad \text { for a.a. } t \in I \text { and all } y \in[\gamma(t)-\varepsilon, \gamma(t)+\varepsilon] \text {, }
$$

or

$$
\gamma^{\prime}(t)-\psi(t)>f(t, y) \quad \text { for a.a. } t \in I \text { and all } y \in[\gamma(t)-\varepsilon, \gamma(t)+\varepsilon]
$$

We say that the admissible discontinuity curve $\gamma$ is inviable for the differential equation if it satisfies (1.3) or (1.4).

The following notation and proposition are useful to check condition (1.2) in Theorem 1.1: for a given operator $T: K \longrightarrow K$ in the conditions of Theorem 1.1 we define

$$
\mathbb{T} x=\bigcap_{\varepsilon>0} \overline{\operatorname{co}} T\left(B_{\varepsilon}(x) \cap K\right) \quad(x \in K) .
$$

Notice that $\mathbb{T} x=\{T x\}$ when $T$ is continuous at $x \in K$. Moreover, the definition of $\mathbb{T}$ can be expressed analytically as follows.

Proposition 1.2 In the conditions of Theorem 1.1, let $x, y \in K$ be fixed.

The following two statements are equivalent:

1. $y \in \mathbb{T} x$ as defined in (1.5).

2. For every $\varepsilon>0$ and every $\rho>0$ there exists a finite family of vectors $x_{i} \in B_{\varepsilon}(x) \cap K$ and coefficients $\lambda_{i} \in[0,1](i=1,2, \ldots, m)$ such that $\sum \lambda_{i}=1$ and

$$
\left\|y-\sum_{i=1}^{m} \lambda_{i} T x_{i}\right\|_{X}<\rho .
$$

\section{Main result}

This section is devoted to the proof of the following existence principle. Notice that $f$ need not be continuous with respect to any of its arguments.

Theorem 2.1 Let $R \in(0, \infty)$ be fixed. Problem (1.1) has at least one absolutely continuous solution $x: I \longrightarrow \mathbb{R}$ such that $\|x\|_{\infty} \leq R$, provided that $F: \mathcal{C}(I) \longrightarrow \mathbb{R}$ is continuous and the following conditions are satisfied:

(H1) There exist $N \geq 0$ and $M \in L^{1}(I,[0, \infty))$ such that $N+\|M\|_{1} \leq R,|F(x)| \leq N$ if $\|x\|_{\infty} \leq R$, and for a.a. $t \in I$ and all $x \in[-R, R]$ we have $|f(t, x)| \leq M(t)$. 
(H2) The compositions $t \in I \mapsto f(t, x(t))$ are measurable if $x \in \mathcal{C}(I)$ and $\|x\|_{\infty} \leq R$.

(H3) There exist admissible discontinuity curves $\gamma_{n}: I_{n}=\left[a_{n}, b_{n}\right] \longrightarrow \mathbb{R}(n \in \mathbb{N})$ such that for a.a. $t \in I$ the function $x \mapsto f(t, x)$ is continuous at every $x \in[-R, R] \backslash \bigcup_{\left\{n: t \in I_{n}\right\}}\left\{\gamma_{n}(t)\right\}$.

Proof Consider the Banach space $X=\mathcal{C}(I)$ with the sup-norm $\|\cdot\|_{\infty}$. In the convex subset

$$
K=\left\{x \in \mathcal{C}(I):\left|x\left(t_{0}\right)\right| \leq N,|x(t)-x(s)| \leq \int_{s}^{t} M(r) d r(s \leq t)\right\},
$$

we define a fixed point operator $T: K \longrightarrow K$ by

$$
T x(t)=F(x)+\int_{t_{0}}^{t} f(s, x(s)) d s \quad(t \in I, x \in K) .
$$

Notice that, thanks to conditions (H1) and (H2), $K$ is a compact subset of the ball $\{x \in$ $\left.\mathcal{C}(I):\|x\|_{\infty} \leq R\right\}$ and $T$ is well-defined and maps $K$ into itself. Therefore it only remains to prove that condition (1.2) in Theorem 1.1 is satisfied. Let $x \in K$ be fixed; we have to prove that $\mathbb{T} x \cap\{x\} \subset\{T x\}$, where $\mathbb{T}$ is as in (1.5).

Case $1-m\left(\left\{t \in I_{n}: x(t)=\gamma_{n}(t)\right\}\right)=0$ for all $n \in \mathbb{N}$. Let us prove that then $T$ is continuous at $x$, which implies that $\mathbb{T} x=\{T x\}$, and then (1.2) is satisfied.

The assumption implies that for a.a. $t \in I$ the mapping $f(t, \cdot)$ is continuous at $x(t)$. Hence if $x_{k} \rightarrow x$ in $K$ then $F\left(x_{k}\right) \rightarrow F(x)$, and

$$
f\left(t, x_{k}(t)\right) \rightarrow f(t, x(t)) \quad \text { for a.a. } t \in I,
$$

which, along with (H1), yield $T x_{k} \rightarrow T x$ uniformly on $I$.

Case $2-m\left(\left\{t \in I_{n}: x(t)=\gamma_{n}(t)\right\}\right)>0$ for some $n \in \mathbb{N}$ such that $\gamma_{n}$ is inviable. In this case we can prove that $x \notin \mathbb{T} x$, and so (1.2) obtains.

First, we fix some notation. Let us assume that for some $n \in \mathbb{N}$ we have $m\left(\left\{t \in I_{n}: x(t)=\right.\right.$ $\left.\left.\gamma_{n}(t)\right\}\right)>0$ and there exist $\varepsilon>0$ and $\psi \in L^{1}\left(I_{n}\right), \psi(t)>0$ for a.a. $t \in I_{n}$, such that (1.4) holds with $\gamma$ replaced by $\gamma_{n}$ (The proof is similar if we assume (1.3) instead of (1.4), so we omit it.).

We denote $J=\left\{t \in I_{n}: x(t)=\gamma_{n}(t)\right\}$, and we deduce from [1], Lemma 4.1, that there is a measurable set $J_{0} \subset J$ with $m\left(J_{0}\right)=m(J)>0$ such that for all $\tau_{0} \in J_{0}$ we have

$$
\lim _{t \rightarrow \tau_{0}^{+}} \frac{2 \int_{\left[\tau_{0}, t\right] \bigvee} M(s) d s}{(1 / 4) \int_{\tau_{0}}^{t} \psi(s) d s}=0=\lim _{t \rightarrow \tau_{0}^{-}} \frac{2 \int_{\left[t, \tau_{0}\right] \bigvee} M(s) d s}{(1 / 4) \int_{t}^{\tau_{0}} \psi(s) d s} .
$$

By [1], Corollary 4.3, there exists $J_{1} \subset J_{0}$ with $m\left(J_{0} \backslash J_{1}\right)=0$ such that for all $\tau_{0} \in J_{1}$ we have

$$
\lim _{t \rightarrow \tau_{0}^{+}} \frac{\int_{\left[\tau_{0}, t\right] \cap J_{0}} \psi(s) d s}{\int_{\tau_{0}}^{t} \psi(s) d s}=1=\lim _{t \rightarrow \tau_{0}^{-}} \frac{\int_{\left[t, \tau_{0}\right] \cap J_{0}} \psi(s) d s}{\int_{t}^{\tau_{0}} \psi(s) d s} .
$$

Let us now fix a point $\tau_{0} \in J_{1}$. From (2.2) and (2.3) we deduce that there exist $t_{-}<\tau_{0}$ and $t_{+}>\tau_{0}, t_{ \pm}$sufficiently close to $\tau_{0}$ so that the following inequalities are satisfied:

$$
2 \int_{\left[\tau_{0}, t_{+}\right] \bigvee} M(s) d s<\frac{1}{4} \int_{\tau_{0}}^{t_{+}} \psi(s) d s,
$$




$$
\begin{aligned}
& \int_{\left[\tau_{0}, t_{+}\right] \cap J} \psi(s) d s \geq \int_{\left[\tau_{0}, t_{+}\right] \cap J_{0}} \psi(s) d s>\frac{1}{2} \int_{\tau_{0}}^{t_{+}} \psi(s) d s, \\
& 2 \int_{\left[t_{-}, \tau_{0}\right] \bigvee} M(s) d s<\frac{1}{4} \int_{t_{-}}^{\tau_{0}} \psi(s) d s, \\
& \int_{\left[t_{-}, \tau_{0}\right] \cap J_{0}} \psi(s) d s>\frac{1}{2} \int_{t_{-}}^{\tau_{0}} \psi(s) d s .
\end{aligned}
$$

Finally, we define a positive number

$$
\rho=\min \left\{\frac{1}{4} \int_{t_{-}}^{\tau_{0}} \psi(s) d s, \frac{1}{4} \int_{\tau_{0}}^{t_{+}} \psi(s) d s\right\}
$$

and we are now in a position to prove that $x \notin \mathbb{T} x$. By virtue of Proposition 1.2, it suffices to prove the following claim.

Claim - Let $\varepsilon>0$ be given by our assumptions over $\gamma_{n}$ and let $\rho$ be as in (2.8). For every finite family $x_{i} \in B_{\varepsilon}(x) \cap K$ and $\lambda_{i} \in[0,1](i=1,2, \ldots, m)$, with $\sum \lambda_{i}=1$, we have $\| x-$ $\sum \lambda_{i} T x_{i} \|_{\infty} \geq \rho$.

Let us denote $y=\sum \lambda_{i} T x_{i}$. For a.a. $t \in I$ we have

$$
y^{\prime}(t)=\sum_{i=1}^{m} \lambda_{i}\left(T x_{i}\right)^{\prime}(t)=\sum_{i=1}^{m} \lambda_{i} f\left(t, x_{i}(t)\right) \leq M(t) .
$$

On the other hand, for every $t \in J=\left\{t \in I_{n}: x(t)=\gamma_{n}(t)\right\}$, we have

$$
\left|x_{i}(t)-\gamma_{n}(t)\right|=\left|x_{i}(t)-x(t)\right|<\varepsilon
$$

and then the assumptions on $\gamma_{n}$ ensure that for a.a. $t \in J$ we have

$$
y^{\prime}(t)=\sum_{i=1}^{m} \lambda_{i} f\left(t, x_{i}(t)\right)<\sum_{i=1}^{m} \lambda_{i}\left(\gamma_{n}^{\prime}(t)-\psi(t)\right)=\gamma_{n}^{\prime}(t)-\psi(t) .
$$

Well-known results, e.g. [5], Lemma 6.92, guarantee that $\gamma_{n}^{\prime}(t)=x^{\prime}(t)$ for a.a. $t \in J$, hence

$$
y^{\prime}(t)<x^{\prime}(t)-\psi(t) \text { for a.a. } t \in J
$$

Now we use (2.10) and (2.9) to deduce the following estimate:

$$
\begin{aligned}
y\left(\tau_{0}\right)-y\left(t_{-}\right)= & \int_{t_{-}}^{\tau_{0}} y^{\prime}(s) d s=\int_{\left[t_{-}, \tau_{0}\right] \cap J} y^{\prime}(s) d s+\int_{\left[t_{-}, \tau_{0}\right] \bigvee} y^{\prime}(s) d s \\
& <\int_{\left[t_{-}, \tau_{0}\right] \cap J}\left(x^{\prime}(s)-\psi(s)\right) d s+\int_{\left[t_{-}, \tau_{0}\right] \bigvee} M(s) d s \\
= & x\left(\tau_{0}\right)-x\left(t_{-}\right)-\int_{\left[t_{-}, \tau_{0}\right] \bigvee} x^{\prime}(s) d s-\int_{\left[t_{-}, \tau_{0}\right] \cap J} \psi(s) d s \\
& +\int_{\left[t_{-}, \tau_{0}\right] \bigvee} M(s) d s \\
< & x\left(\tau_{0}\right)-x\left(t_{-}\right)-\int_{\left[t_{-}, \tau_{0}\right] \cap} \psi(s) d s+2 \int_{\left[t_{-}, \tau_{0}\right] \bigvee} M(s) d s
\end{aligned}
$$




$$
\begin{aligned}
& \leq x\left(\tau_{0}\right)-x\left(t_{-}\right)-\int_{\left[t_{-}, \tau_{0}\right] \cap J_{0}} \psi(s) d s+2 \int_{\left[t_{-}, \tau_{0}\right] \bigvee} M(s) d s \\
& <x\left(\tau_{0}\right)-x\left(t_{-}\right)-\frac{1}{4} \int_{t_{-}}^{\tau_{0}} \psi(s) d s \quad \text { (by (2.6) and (2.7)). }
\end{aligned}
$$

Hence $\|x-y\|_{\infty} \geq y\left(t_{-}\right)-x\left(t_{-}\right) \geq \rho$ provided that $y\left(\tau_{0}\right) \geq x\left(\tau_{0}\right)$.

Similar computations with $t_{+}$instead of $t_{-}$show that if $y\left(\tau_{0}\right) \leq x\left(\tau_{0}\right)$ then we also have $\|x-y\|_{\infty} \geq \rho$.

Case $3-m\left(\left\{t \in I_{n}: x(t)=\gamma_{n}(t)\right\}\right)>0$ only for some of those $n \in \mathbb{N}$ such that $\gamma_{n}$ is viable. Let us prove that in this case the relation $x \in \mathbb{T} x$ implies $x=T x$.

We lose no generality if we assume that all admissible discontinuity curves are viable and $m\left(J_{n}\right)>0$ for all $n \in \mathbb{N}$, where

$$
J_{n}=\left\{t \in I_{n}: x(t)=\gamma_{n}(t)\right\}
$$

For each $n \in \mathbb{N}$ and for a.a. $t \in J_{n}$ we have

$$
x^{\prime}(t)=\gamma_{n}^{\prime}(t)=f\left(t, \gamma_{n}(t)\right)=f(t, x(t))
$$

and therefore $x^{\prime}(t)=f(t, x(t))$ a.e. in $A=\bigcup_{n \in \mathbb{N}} J_{n}$.

Now we assume that $x \in \mathbb{T} x$ and we prove that it implies that $x^{\prime}(t)=f(t, x(t))$ a.e. in $I \backslash A$, and that $x\left(t_{0}\right)=F(x)$, thus showing that $x=T x$.

Since $x \in \mathbb{T} x$ then for each $k \in \mathbb{N}$ we can use Proposition 1.2 with $\varepsilon=\rho=1 / k$ to guarantee that we can find functions $x_{k, i} \in B_{1 / k}(x) \cap K$ and coefficients $\lambda_{k, i} \in[0,1](i=1,2, \ldots, m(k))$ such that $\sum_{i} \lambda_{k, i}=1$ and

$$
\left\|x-\sum_{i=1}^{m(k)} \lambda_{k, i} T x_{k, i}\right\|_{\infty}<\frac{1}{k}
$$

Let us denote $y_{k}=\sum_{i=1}^{m(k)} \lambda_{k, i} T x_{k, i}$, and notice that $y_{k} \rightarrow x$ uniformly in $I$ and $\left\|x_{k, i}-x\right\| \leq$ $1 / k$ for all $k \in \mathbb{N}$ and all $i \in\{1,2, \ldots, m(k)\}$.

On the other hand, for a.a. $t \in I \backslash A$ we see that $f(t, \cdot)$ is continuous at $x(t)$ so for any $\varepsilon>0$ there is some $k_{0}=k_{0}(t) \in \mathbb{N}$ such that for all $k \in \mathbb{N}, k \geq k_{0}$, we have

$$
\left|f\left(t, x_{k, i}(t)\right)-f(t, x(t))\right|<\varepsilon \quad \text { for all } i \in\{1,2, \ldots, m(k)\}
$$

and therefore

$$
\left|y_{k}^{\prime}(t)-f(t, x(t))\right| \leq \sum_{i=1}^{m(k)} \lambda_{k, i}\left|f\left(t, x_{k, i}(t)\right)-f(t, x(t))\right|<\varepsilon
$$

Hence $y_{k}^{\prime}(t) \rightarrow f(t, x(t))$ for a.a. $t \in I \backslash A$, and then we conclude from [1], Corollary 4.3, that $x^{\prime}(t)=f(t, x(t))$ for a.a. $t \in I \backslash A$.

Finally, to prove that $x\left(t_{0}\right)=F(x)$ we fix $\rho>0$ and, since $F$ is continuous, there exists $\varepsilon>0$ such that

$$
|F(y)-F(x)|<\rho / 2 \quad \text { for all } y \in B_{\varepsilon}(x) .
$$


Since $x \in \mathbb{T} x$, we know from Proposition 1.2 that we can find functions $x_{i} \in B_{\varepsilon}(x) \cap K$ and coefficients $\lambda_{i} \in[0,1](i=1,2, \ldots, m)$ such that $\sum_{i} \lambda_{i}=1$ and

$$
\left\|x-\sum_{i=1}^{m} \lambda_{i} T x_{i}\right\|_{\infty}<\rho / 2 .
$$

The definition of $T$ ensures that $\sum \lambda_{i} T x_{i}\left(t_{0}\right)=\sum \lambda_{i} F\left(x_{i}\right)$, so (2.12) implies that

$$
\left|x\left(t_{0}\right)-\sum_{i=1}^{m} \lambda_{i} F\left(x_{i}\right)\right|<\rho / 2 .
$$

Using (2.13) and (2.11), we deduce that

$$
\begin{aligned}
\left|x\left(t_{0}\right)-F(x)\right| & \leq\left|x\left(t_{0}\right)-\sum_{i=1}^{m} \lambda_{i} F\left(x_{i}\right)\right|+\left|\sum_{i=1}^{m} \lambda_{i} F\left(x_{i}\right)-F(x)\right| \\
& <\frac{\rho}{2}+\left|\sum_{i=1}^{m} \lambda_{i}\left[F\left(x_{i}\right)-F(x)\right]\right|<\frac{\rho}{2}+\frac{\rho}{2}=\rho .
\end{aligned}
$$

This proves that $x\left(t_{0}\right)=F(x)$ because $\rho>0$ can be chosen as small as we wish.

\section{Examples and nonexistence of extremal solutions}

Given $n \in \mathbb{N}$, we define $\phi(1)=2$ and $\phi(n)$ as the number of divisors of $n$ if $n \geq 2$. Thus constructed, $\phi$ is an unbounded sequence satisfying that $\phi(n) \geq 2$ for all $n \in \mathbb{N}$ and, as there exist infinitely many prime numbers,

$$
\liminf _{n \rightarrow \infty} \phi(n)=2
$$

Now we consider the function

$$
f(t, x)=\frac{1}{\phi(n) \sqrt{|t|}}+\frac{1}{2}, \quad t \neq 0, x \in \mathbb{R}
$$

where $n \in \mathbb{N}$ is chosen such that

$$
\frac{n-1}{t} \leq x<\frac{n}{t}, \quad \text { if } t x \geq 0, \quad-(n-1)^{\sigma} t \leq x<-n^{\sigma} t, \quad \sigma=\operatorname{sgn} t, \text { if } t x<0 .
$$

We will show that Theorem 2.1 can be applied to guarantee the existence of solutions for the following multipoint problem:

$$
\left\{\begin{array}{l}
x^{\prime}(t)=f(t, x(t)) \quad \text { for a.a. } t \in I=[-1,1] \\
x(0)=\sum_{i=1}^{5} p_{i}\left(x\left(t_{i}\right)\right)
\end{array}\right.
$$

where $t_{i} \in I$ for $i=1, \ldots, 5$, and the functions $p_{i}: \mathbb{R} \longrightarrow \mathbb{R}$ are continuous and satisfy

$$
\left|p_{i}(x)\right| \leq \frac{2}{5} \quad \text { for all } x \in[-5,5]
$$


Proposition 3.1 Problem (3.2), with $f(t, x)$ defined in (3.1), has at least one absolutely continuous solution $x$ such that $\|x\|_{\infty} \leq 5$.

Proof First, notice that we can write the multipoint condition in the form $x(0)=F(x)$ for

$$
F(x)=\sum_{i=1}^{5} p_{i}\left(x\left(t_{i}\right)\right)
$$

and $F: \mathcal{C}(I) \longrightarrow \mathbb{R}$ is continuous.

Now we take $R=5$ and we note that if $x \in \mathcal{C}(I)$ and $\|x\|_{\infty} \leq R$, then $|F(x)| \leq N=2$. On the other hand, since $\phi(n) \geq 2$ for all $n \in \mathbb{N}$, then for a.a. $t \in I$ and all $x \in[-5,5]$ we have

$$
|f(t, x)|=\frac{1}{\phi(n) \sqrt{|t|}}+\frac{1}{2} \leq \frac{1}{2 \sqrt{|t|}}+\frac{1}{2}=M(t) .
$$

Since

$$
\int_{-1}^{1} M(t) d t=3
$$

condition (H1) is satisfied.

To check condition (H2), notice that for every $x \in \mathcal{C}(I)$ we can write the composition $t \in I \longmapsto f(t, x(t))$ as

$$
t \longmapsto f(t, x(t))=\sum_{n=1}^{\infty}\left(\frac{1}{\phi(n) \sqrt{|t|}}+\frac{1}{2}\right)\left(\chi_{I_{n}}(t) \chi_{I_{n}}(t)+\chi_{\hat{I}_{n}}(t) \chi_{\hat{J}_{n}}(t)\right),
$$

with

$$
\begin{aligned}
& I_{n}=x^{-1}\left[\frac{n-1}{t}, \frac{n}{t}\right), \quad J_{n}=\{t \in I: \operatorname{sgn} t=\operatorname{sgn} x(t)\}, \\
& \hat{I}_{n}=x^{-1}[-(n-1) t,-n t), \quad \hat{J}_{n}=\{t \in I: \operatorname{sgn} t=-\operatorname{sgn} x(t)\},
\end{aligned}
$$

and so $f(\cdot, x(\cdot))$ is a measurable function.

Finally, to check condition (H3) notice that definition of $\phi$ implies that for a.a. $t \in I$ there exists a countable set $K \subset \mathbb{N}$ such that $f(t, \cdot)$ is discontinuous in $\bigcup_{k \in K} \gamma_{k}(t)$ and $\bigcup_{k \in K} \hat{\gamma}_{k}(t)$, with

$$
\gamma_{k}(t)=\frac{k}{t}, \quad \hat{\gamma}_{k}(t)=-k^{\sigma} t
$$

We have $\gamma_{k}^{\prime}(t)<0$ and $\hat{\gamma}_{k}^{\prime}(t)<0$ for a.a. $t \in I$ and all $k \in K$; however, $f(t, u) \geq \frac{1}{2}$ for a.a. $t \in I$ and all $u \in \mathbb{R}$, and so the discontinuity curves are inviable for the differential equation.

We can conclude by application of Theorem 2.1 that problem (3.2) has at least one absolutely continuous solution $x$, which, moreover, satisfies $\|x\|_{\infty} \leq 5$.

In the next example we show that, in general, there is no hope to have extremal solutions of (1.1) in the conditions of Theorem 2.1. By extremal solutions we mean a pair of solutions $x_{*}$ and $x^{*}$ (possibly identical) such that any other solution $x$ of (1.1) satisfies $x_{*}(t) \leq x(t) \leq$ $x^{*}(t)$ for all $t \in I$. 
Example 3.1 Consider the following particular case of (1.1):

$$
\begin{aligned}
& x^{\prime}=f(t, x)=\left\{\begin{array}{ll}
1, & \text { if } t x>0, \\
-1, & \text { if } t x<0, \\
0, & \text { if } t x=0
\end{array}\right\} \text { for a.a. } t \in I=[-1,1], \\
& x(0)=F(x)=\frac{x(-1)+x(1)}{7} .
\end{aligned}
$$

It is easy to show that problem (3.3)-(3.4) satisfies conditions (H1) and (H2) in Theorem 2.1. Indeed, for (H1) it suffices to take $R=3, N=6 / 7$, and $M(t)=1$ for all $t \in I$. Condition (H3) is equally easy to check: we only need one admissible discontinuity curve, namely, $\gamma(t)=0$ for all $t \in I$, which is also a solution of the problem (3.3)-(3.4) and, in particular, a viable discontinuity curve for (3.3).

Other solutions of (3.3)-(3.4) are, obviously, $x_{ \pm}(t)= \pm t$ for $t \in[-1,1]$. The remaining solutions can be obtained using the general solution of the differential equation (3.3) and then imposing the initial condition (3.4). In doing so, we find that the set of solutions of (3.3)-(3.4) is the uniparametric family

$$
x_{\tau}(t)=\left\{\begin{array}{ll}
t+\tau, & \text { if } t \in[-1,-\tau], \\
0, & \text { if } t \in(-\tau, \tau), \\
t-\tau, & \text { if } t \in[\tau, 1],
\end{array} \quad \text { for each } \tau \in[0,1]\right.
$$

Notice that there is not a greatest or a least element in $\left\{x_{\tau}: \tau \in[0,1]\right\}$ with respect to the usual pointwise partial order. Summing up, we have an example of a problem under the conditions of Theorem 2.1 which lacks extremal solutions.

\section{Comparison with recent literature}

Theorem 2.1 complements some recent existence results. Pikuta and Rzymowski [6] proved that the problem

$$
x^{\prime}(t)=f(x(t))+h(t) \quad \text { for a.a. } t \geq 0, \quad x(0)=0,
$$

has absolutely continuous local solutions provided that

(C1) there exists $M>0$ such that $0<f(x)<+\infty$ for a.a. $x \in[0, M]$ and $\int_{0}^{M} \frac{d x}{f(x)}<+\infty$;

(C2) $h:[0,+\infty) \rightarrow[0,+\infty]$ is locally integrable.

Uniqueness of solutions for (4.1) is studied in [7] under similar conditions for $f$ and assuming that $h$ is of bounded variation.

In this section we show that Theorem 2.1 guarantees existence of solutions to some cases of (4.1) not covered by the existence results in $[6,7]$.

Let us consider the discontinuous even function $\phi: \mathbb{R} \longrightarrow \mathbb{R}$ defined by $\phi(0)=0, \phi(x)=$ $1 /(n+1)$ if $1 /(n+1) \leq x<1 / n$ for some $n \in \mathbb{N}$, and $\phi(x)=1$ for $x \geq 1$.

Now we fix $\delta>0, h \in L^{1}(-\delta, \delta), h(t)>0$ for a.a. $t \in(-\delta, \delta)$, and we consider the initial value problem

$$
x^{\prime}=|x+\phi(x)|+h(t), \quad x(0)=0 .
$$


Notice that (4.2) is the particular case of (4.1) corresponding to $f(x)=|x+\phi(x)|$. Since $0 \leq \phi(x) \leq x$ for all $x \geq 0$, we have

$$
\int_{0}^{M} \frac{d x}{f(x)}=+\infty
$$

for all $M>0$. Therefore, $f$ does not satisfy the condition $(\mathrm{C} 1)$ and, as a result, problem (4.2) falls outside the scope of the existence results in $[6,7]$.

However, Theorem 2.1 ensures the existence of solutions of (4.2).

Proposition 4.1 If $\delta \in(0,1 / 4)$, then (4.2) has at least one solution defined on the interval $[-\delta, \delta]$.

Proof We note that (4.2) is the particular case of (1.1) corresponding to $f(t, x)=|x+\phi(x)|+$ $h(t), t_{0}=0, L=\delta$, and $F(x)=0$ for all $x \in \mathcal{C}(I), I=[-\delta, \delta]$.

Since $1-4 \delta>0$, we can take $R>0$ such that

$$
\frac{\int_{-\delta}^{\delta} h(t) d t}{1-4 \delta} \leq R
$$

For each $x \in[-R, R]$ we have

$$
|f(t, x)| \leq|x|+\phi(x)+h(t) \leq 2 R+h(t)=: M(t)
$$

and then condition (H1) in Theorem 2.1 is satisfied for this choice of $M(t), R$ as in (4.3), and $N=0$.

For every $x \in \mathcal{C}(I)$ the sets $\{t \in I: 1 /(n+1) \leq|x(t)|<1 / n\}, n \in \mathbb{N}$, are measurable and $\phi(x(t))$ is constant on those sets. Hence $f(\cdot, x(\cdot))$ is measurable.

Finally, for almost all $t \in I$ the mapping $x \in \mathbb{R} \longmapsto f(t, x)$ is continuous in $\mathbb{R} \backslash\{ \pm 1 / n: n \in$ $\mathbb{N}\}$, and each function

$$
\gamma_{ \pm n}(t)= \pm \frac{1}{n} \quad(t \in I, n \in \mathbb{N})
$$

is an inviable discontinuity curve because $f(t, y)>h(t)$ for every $y \neq 0$ and $h(t)>0$ almost everywhere.

Since all the conditions in Theorem 2.1 are satisfied, we conclude that (4.2) has at least one solution $x:[-\delta, \delta] \rightarrow \mathbb{R}$.

\section{Competing interests}

The authors declare that they have no competing interests.

Authors' contributions

Both authors have contributed equally to this paper. Both authors read and approved the final manuscript.

\section{Acknowledgements}

The first author is partially supported by Xunta de Galicia, Consellería de Cultura, Educación e Ordenación Universitaria, through the project EM2014/032 'Ecuacións diferenciais non lineares. The second author is partially supported by Ministerio de Economía y Competitividad, Spain, and FEDER, Projects MTM2010-15314 and MTM2013-43014-P. 
References

1. López Pouso, R: Schauder's fixed-point theorem: new applications and a new version for discontinuous operators. Bound. Value Probl. 2012, Article ID 92 (2012)

2. Biles, DC, López Pouso, R: First-order singular and discontinuous differential equations. Bound. Value Probl. 2009, Article ID 507671 (2009)

3. Cid, JÁ, Pouso, RL: Ordinary differential equations and systems with time-dependent discontinuity sets. Proc. R. Soc. Edinb., Sect. A 134(4), 617-637 (2004)

4. López Pouso, R: On the Cauchy problem for first order discontinuous ordinary differential equations. J. Math. Anal. Appl. 264(1), 230-252 (2001)

5. Stromberg, KR: An Introduction to Classical Real Analysis. Wadsworth, Belmont (1981)

6. Pikuta, P, Rzymowski, W: Non-autonomous scalar discontinuous ordinary differential equation. J. Math. Anal. Appl. 307(1), 496-503 (2005)

7. Cid, JÁ, Pouso, RL: Existence of unique solutions for perturbed autonomous differential equations. J. Lond. Math. Soc (2) $78,798-812(2008)$

Submit your manuscript to a SpringerOpen ${ }^{\circ}$ journal and benefit from:

- Convenient online submission

Rigorous peer review

- Immediate publication on acceptance

- Open access: articles freely available online

- High visibility within the field

- Retaining the copyright to your article 